Vietnam Journal of Mechanics, VAST, Vol.39, No. 2 (2017), pp. 165-176

DOI:10.15625/0866-7136/8760

\title{
NEW TEMPERATURE JUMP BOUNDARY CONDITION IN HIGH-SPEED RAREFIED GAS FLOW SIMULATIONS
}

\author{
Nam T. P. Le ${ }^{1, *}$, Ngoc Anh Vu ${ }^{2}$, Le Tan Loc ${ }^{3}$, Tran Ngoc Thoai ${ }^{4}$ \\ ${ }^{1}$ Institute for Computational Science, Ton Duc Thang University, Ho Chi Minh City, Vietnam \\ ${ }^{2}$ Ho Chi Minh City University of Technology, VNU-HCM, Vietnam \\ ${ }^{3}$ Vietnam Aviation Academy, Ho Chi Minh City, Vietnam \\ ${ }^{4}$ Industrial University of Ho Chi Minh City, Vietnam \\ *E-mail: letuanphuongnam@tdt.edu.vn \\ Received October 05, 2016
}

\begin{abstract}
The effect of the sliding friction has been important in calculating the heat flux of gas flow from the surface since there is some slip over the surface. There has not been any the temperature jump condition including the sliding friction part so far. In this paper, we will propose a new temperature jump condition that includes the sliding friction. Our new temperature jump condition will be evaluated for NACA0012 micro-airfoil in highspeed rarefied gas flow simulations using the CFD method, which solves the NavierStokes equations within the OpenFOAM framework with working gas as air. The airfoil case is simulated with various Knudsen numbers from 0.026 to 0.26 , and the angles-ofattack (AOAs) from 0-deg to 20-deg. The surface gas temperatures predicting by our new temperature jump condition give good agreements with the DSMC data, especially the NACA0012 micro-airfoil cases with the high Knudsen numbers, $K n=0.1$, and $K n=0.26$ with $\mathrm{AOA}=20-\mathrm{deg}$. for the lower surface.
\end{abstract}

Keywords: Sliding friction, new temperature jump condition, NACA0012 airfoil.

\section{INTRODUCTION}

The simulation of nonequilibrium hypersonic gas flow is important for the aerodynamic design of space and re-entry vehicles. These flows are most successfully simulated by the Direct Simulation Monte Carlo (DSMC) method; however, the computational cost of this method is expensive in comparison to Computational Fluid Dynamics (CFD), which solves the Navier-Stokes-Fourier (NSF) equations. The present work focuses on investigating the temperature jump boundary condition for use with the NSF equations applied to rarefied gas flow simulations. Translational nonequilibrium of a rarefied gas flow can be characterized by the Knudsen number, $K n$, that is the ratio of the molecular mean free path, $\lambda$, to the macroscopic characteristic length, $L$. When $K n \leq 0.001$ that is

(c) 2017 Vietnam Academy of Science and Technology 
continuum regime, and in the range of $0.001<K n<0.1$, that is called slip regime. $\mathrm{Kn}$ rises into the range $0.1 \leq K n<10$, that is called the transition regime. For $K n \geq 10$, the flow is called to be in the free molecular regime. The NSF equations with velocity slip and temperature jump boundary conditions can capture the rarefied gas flow in the range of $0.001<K n<0.1$, i.e. slip regime. However, as $K n$ rises into the range $0.1 \leq K n<10$ (transition regime), the NSF equations become inappropriate because the near-equilibrium fluid assumption for flows has broken down.

The design of hypersonic vehicles requires accurate prediction of the surface properties in flight. One of them is typically the surface gas temperature or heat flux. The peak surface temperatures or heat fluxes are integrated over time and mapped over the vehicle surface as part of the process to design the thermal protection system. The temperature jump condition was an early development by Smoluchowski [1], and was driven by the heat flux to the surface in the normal direction. Thereafter, several temperature jump conditions were developed in [2-9] for CFD to improve the simulation results of the surface gas temperature in the slip regime $(0.01 \leq K n \leq 0.1)$.

In the literature, all derivations of the temperature jump condition have not been considered the sliding friction. It was first introduced by Maslen [10] in calculating the heat transfer of gas flows over the surface. He stated that his formulation is correct for heat transfer without further proof. However, the sliding friction term could be directly derived with kinetic theory for a nonuniform gas, and was presented in $[11,12]$. The heat transfer generating by the sliding friction was computed based on the slip velocity and shear stress. It means if there is some slip of gas flows over the surface then the sliding friction should be considered. In CFD, we have often used the slip velocity and temperature jump conditions to improve the NSF equations in the slip regime $(0.01 \leq K n \leq 0.1)$. There was a different nature in computation of the surface gas temperature (or temperature jump) between the DSMC and CFD methods. In DSMC, it was calculated as a function of velocity components $[13,14]$, while that in CFD was computed by the normal gradient of temperature only (Fourier's law). This leads to a difference between the DSMC and CFD surface gas temperatures. Therefore, the sliding friction needs to be taken into account in developing the temperature jump condition to improve the prediction of surface gas temperature in CFD.

In the present work we will propose a new temperature jump condition by considering the sliding friction at the wall. Our new temperature jump condition will be implemented in OpenFOAM [15] to employ with the solver "rhoCentralFoam" for CFD simulations. OpenFOAM is an open source CFD toolbox, written in C++. All solvers developed within OpenFOAM are, by default, 3-dimensional, but can be used for 1- or 2-dimensional problems by the application of particular boundary conditions lying on the plane of directions of no interest. Meanwhile the heat transfers involving the sliding friction proposed by Maslen [10] and without the sliding friction (i.e. the Fourier law of heat conduction) are also compared with DSMC data. Micro-airfoil NACA0012 is chosen to evaluate our new temperature jump condition with Mach number, $M=2$, various Knudsen numbers, $K n$, from 0.026 to 0.26 and angles-of-attack (AOAs) from 10-deg. to 20-deg., [16-18]. NACA0012 airfoil is basic configuration for testing the new boundary 
conditions. We also investigate whether the surface gas temperatures with our new temperature jump condition can capture those of DSMC data of the airfoil cases with $\mathrm{Kn}=$ 0.1 and 0.26 (i.e. in the transition regime).

\section{NONEQUILIBRIUM BOUNDARY CONDITIONS}

\subsection{Smoluchowski/Maxwell boundary conditions in CFD}

As our new temperature jump condition is derived from the Smoluchowski theory, so the first order Maxwell/Smoluchowski slip velocity and temperature jump boundary conditions are adopted for comparison. The first order Maxwell slip velocity condition is expressed as follows [19]

$$
\mathbf{u}+\left(\frac{2-\sigma_{u}}{\sigma_{u}}\right) \lambda\left(\mathbf{S} \cdot \nabla_{n} \mathbf{u}\right)=\mathbf{u}_{w}-\left(\frac{2-\sigma_{u}}{\sigma_{u}}\right) \frac{\lambda}{\mu} \mathbf{S} \cdot\left(\mathbf{n} \cdot \boldsymbol{\Pi}_{m c}\right)-\frac{3}{4} \frac{\mu}{\rho} \frac{\mathbf{S} \cdot \nabla T}{T},
$$

where $\mathbf{u}$ is the velocity; $\lambda$ is the mean free path; $\gamma$ is the specific heat ratio; $\mu$ is viscosity; $\rho$ is the density; $T$ is the temperature and $\mathbf{u}_{w}$ is the wall velocity. The tangential momentum accommodation coefficient, $\sigma_{u}$, determines the proportion of molecules reflected from the surface specularly (equal to $1-\sigma_{u}$ ) or diffusely (equal to $\sigma_{u}$ ), and $0 \leq \sigma_{u} \leq 1$. The tensor $\Pi_{m c}=\mu\left((\nabla \mathbf{u})^{T}-\frac{2}{3} \mathbf{I} t r(\nabla \mathbf{u})\right)$ is the stress tensor, where the superscript $T$ denotes the transpose and $t r$ indicates the trace. Tensor $\mathbf{S}=\mathbf{I}-\mathbf{n n}$, where $\mathbf{n}$ is the unit normal vector defined as positive in the direction pointing out of the flow domain and $\mathbf{I}$ is identity tensor, removes normal components of any non-scalar field, e. g., velocity, so that slip only occurs in the direction tangential to the surface.The right-hand side of the Eq. (1) contains three terms that are associated with (in order): the surface velocity, the so-called curvature effect, and thermal creep. The tangential momentum accommodation coefficient, $\sigma_{u}$, determines the proportion of molecules reflected from the surface specularly (equal to $1-\sigma_{u}$ ) or diffusely (equal to $\sigma_{u}$ ), and $0 \leq \sigma_{u} \leq 1$.

The temperature jump condition was proposed by Smoluchowski [1], and was driven by the heat flux to the surface in the normal direction

$$
T+\frac{2-\sigma_{T}}{\sigma_{T}} \frac{2 \gamma}{(\gamma+1) \operatorname{Pr}} \lambda \nabla_{n} T=T_{w}
$$

where $T_{w}$ is the surface temperature and Pr is the Prandtl number. The thermal accommodation coefficient, $\sigma_{T}$, is used to ascribe the temperatures of the receding molecules. Specularly reflected molecules recede from the surface with their original incident energy, and diffusely reflected molecules have their temperatures adjusted to those would arise in a mass of gas in equilibrium at the temperature of the surface. Perfect energy exchange between the gas and the solid surface corresponds to $\sigma_{T}=1$, and no energy exchange to $\sigma_{T}=0$.

\subsection{New temperature jump condition in CFD}

The energy of approaching molecules impinging on a unit area of surface is responsible for the heat conducted through the surface. Smoluchowski assumed that the approaching stream of molecules conducted the same level of heat as that within the region of gas some distance away from the boundary. The approaching and receding 
streams contribute to half of the conduction of heat. The conducted heat per unit area is $\mathbf{n} \cdot \mathbf{q}$ (positive inward), and the approaching stream carries a contribution of half that value to heat conduction. Maslen also introduced the sliding friction in [10] to determine heat conduction over a flat plate since there is some slip at the surface. This term was calculated based on the shear tress, $\tau_{x y}$ and slip velocity, $\mathbf{u}_{s}$. The heat conduction generating by the sliding friction (shear work) should be involved in conservation of energy at the surface. It has been considered to contribute to the component of the total conduction of heat at the surface that is due to the approaching molecules [20], seen in Fig. 1.

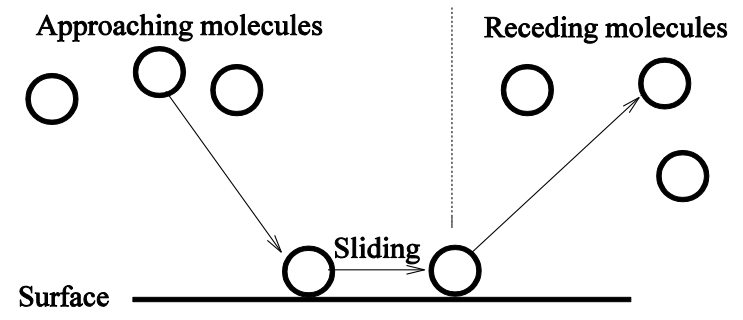

Fig. 1. Schematic of the gas-surface interaction

The shear work per unit area is $-(\mathbf{S} \cdot(\mathbf{n} \cdot \boldsymbol{\Pi})) \cdot\left(\mathbf{u}-\mathbf{u}_{w}\right)$ (positive inwards), where $\mathbf{u}$ is the gas velocity at near the surface; the symbol "." denotes the inner product. In order to determine the temperature jump while conserving energy a thermal accommodation coefficient, $\sigma_{T}$, is introduced (with $0 \leq \sigma_{T} \leq 1$ ). Therefore we have [21]

$$
\text { Heat Flux } x_{\text {total }}=\sigma_{T}\left(\frac{1}{2} \text { Heat Flux } \text { approaching }+\frac{1}{2} \text { Heat Flux } \text { receding }\right) .
$$

The internal energy of the molecules will normally be $e=c_{v} T$ per unit mass, where $c_{v}$ is the specific heat of a gas at constant volume. The energy of colliding molecules is then higher by a factor $(\gamma+1) / 2$, corresponding to an energy $\left(c_{v}+R / 2\right) T$ [21], where $R$ is specific gas constant. The mass of molecules colliding with unit area of boundary is $\rho \bar{v} / 4$ so the integral energy per unit area is $\rho \bar{v} / 4(\gamma+1) e / 8$, where $\bar{v}$ is the mean molecular velocity. The conduction of heat by the receding stream is the difference between the internal energy of the gas $e$ and that of the surface $e_{w}$ and is calculated as [21]

$$
\frac{1}{2} \text { Heat Flux } x_{\text {receding }}=\frac{1}{8} \rho \bar{v}(\gamma+1)\left(e-e_{w}\right),
$$

where $\gamma$ is the specific heat ratio and subscript $w$ denotes the surface. In [12] our previous work illustrated the heat transfer of the slip gas flow at the wall must contain two parts: 1) the conduction heat flux normal to the surface, and 2) the heat flux due to the sliding friction. The simulation results in [12] gave good results for calculating the heat transfer at the wall in comparing with DSMC data. In the Smoluchowski theory developing the temperature jump condition, the heat transfer only considered the conduction heat flux normal to the surface, and omitted the sliding friction part. In CFD solving the NSF equations, the heat transfer of the slip gas flow at the wall is often computed at the post-processing while the surface gas temperature is calculated by the temperature jump 
condition, and updated at each iteration in the computational process. The good prediction of the surface gas temperature will improve the accuracy of the other quantities at the wall in the computational process such as the viscosity, mean-free-path ... The sliding friction term is now included in heat flux at the wall when the viscous heat generation needs to be considered and contribute to the approaching stream

$$
\left[\mathbf{n} \cdot \mathbf{q}-(\mathbf{S} \cdot(\mathbf{n} \cdot \boldsymbol{\Pi})) \cdot\left(\mathbf{u}-\mathbf{u}_{w}\right)\right]=\sigma_{T}\left(\frac{1}{2}\left[\mathbf{n} \cdot \mathbf{q}-(\mathbf{S} \cdot(\mathbf{n} \cdot \boldsymbol{\Pi})) \cdot\left(\mathbf{u}-\mathbf{u}_{w}\right)\right]+\frac{1}{8} \rho \bar{v}(\gamma+1)\left(e-e_{w}\right)\right),
$$

which is re-arranged by replacing

$$
\rho \bar{v}=2 \mu / \lambda
$$

and, $e=c_{v} T$ and $e_{w}=c_{v} T_{w}$ as,

$$
\frac{1}{2} \frac{\mu}{\lambda}(\gamma+1) c_{v}\left(T-T_{w}\right)=\left(\frac{2-\sigma_{T}}{\sigma_{T}}\right)(\mathbf{n} \cdot \mathbf{q})-\left(\frac{2-\sigma_{T}}{\sigma_{T}}\right)\left((\mathbf{S} \cdot(\mathbf{n} \cdot \boldsymbol{\Pi})) \cdot\left(\mathbf{u}-\mathbf{u}_{w}\right)\right),
$$

where $q=-k \nabla T$

$$
\begin{aligned}
\left(T-T_{w}\right)= & -\frac{2 \lambda}{\mu(\gamma+1) c_{v}}\left(\frac{2-\sigma_{T}}{\sigma_{T}}\right)(\mathbf{n} \cdot k \nabla T) \\
& -\left(\frac{2-\sigma_{T}}{\sigma_{T}}\right) \frac{2 \lambda}{\mu(\gamma+1) c_{v}}\left((\mathbf{S} \cdot(\mathbf{n} \cdot \boldsymbol{\Pi})) \cdot\left(\mathbf{u}-\mathbf{u}_{w}\right)\right),
\end{aligned}
$$

with $\mathbf{n} \cdot \nabla T=\nabla_{n} T$, the heat capacity ratio $\gamma=\frac{c_{p}}{c_{v}}$ and thermal conductivity $k=\frac{c_{p} \mu}{\operatorname{Pr}}$, then Eq. (8) is re-arranged as,

$$
T+\frac{\gamma 2 \lambda}{\operatorname{Pr}(\gamma+1)}\left(\frac{2-\sigma_{T}}{\sigma_{T}}\right) \nabla_{n} T=T_{w}-\left(\frac{2-\sigma_{T}}{\sigma_{T}}\right) \frac{2 \lambda}{\mu(\gamma+1) c_{v}}\left((\mathbf{S} \cdot(\mathbf{n} \cdot \boldsymbol{\Pi})) \cdot\left(\mathbf{u}-\mathbf{u}_{w}\right)\right),
$$

where Pr is the Prandtl number and $c_{p}$ is the specific heat of a gas at constant pressure. Eq. (9) is the new temperature jump condition. The second term in the right side is a new term from considering the sliding friction in the temperature jump condition. The Maxwellian mean free path, $\lambda$, and the Sutherland viscosity, $\mu$, are calculated as follows [21]

$$
\lambda=\frac{\mu}{\rho} \sqrt{\frac{\pi}{2 R T}}
$$

and

$$
\mu=A_{S} \frac{T^{1.5}}{T+T_{S}},
$$

where $A_{S}=1.46 \times 10^{-6} \mathrm{~Pa} \mathrm{~s} \mathrm{~K}-1 / 2$ and $T_{S}=110.4 \mathrm{~K}$ for air [5].

The nonequilibrium boundary conditions presented in Eqs. (1), (2) and (9) are implemented in OpenFOAM to work with the solver 'rhoCentralFoam'. The implemented approach of nonequilibrium boundary condition was described in our previous work [5,7]. The Maxwell slip condition with the curvature effect will be used for all CFD simulations. In the solver "rhoCentralFoam" the NSF equations are numerically solved with the high-resolution central scheme described in [22]. 


\subsection{Slip velocity and temperature jump quantities in DSMC}

The slip velocity and temperature jump in DSMC are calculated as post-processing in DSMC based on the gas-surface interactions: DSMC particles are adsorbed on the surface and then re-emitted. In fact, DSMC particles collide with the surface and experience both specular and diffuse interactions. The accommodation coefficients represent the fraction of incoming DSMC particles that are reflected diffusely, and the remainder are reflected specularly. Velocity slip and translational temperature jump are calculated with the accommodation coefficients of unity as presented in [13,14],

$$
\begin{aligned}
& \mathbf{u}_{s}=\frac{\sum\left(\left(m / u_{n}\right) u_{p}\right)}{\sum\left(m / u_{n}\right)}-\mathbf{u}_{w}, \\
& T=\frac{\sum\left(\left(m / u_{n}\right)\|\mathbf{u}\|\right)-\sum\left(m / u_{n}\right) \mathbf{u}_{s}^{2}}{3 k \sum\left(1 / u_{n}\right)}-T_{w} .
\end{aligned}
$$

where $\|\mathbf{u}\|$ is the velocity magnitude. The $u_{n}$ and $u_{p}$ in the equations are taken prior to and after the collision with the surface, and the summations include pre- and postcollision molecules.

It is seen that there is different nature in computation between the temperature jump between the CFD (Eq. (11)) and DSMC (Eq. (13)) formulations. In CFD that is often computed by the temperature gradient normal to the surface only. While the temperature jump has been calculated that as a function of velocities in DSMC. Our simulation results in the previous work [18] figured out the CFD temperature jump could not capture that of DSMC at the high Knudsen numbers, $K n \geq 0.1$. Our new jump condition in Eq. (9) is now included the terms of slip velocity and shear stress (i.e. the velocity gradients) so that it can capture the temperature jump of the high $K n$ cases in comparing with DSMC data.

\section{NUMERICAL SETUPS}

The freestream gas flow conditions and working gas of micro-airfoil NACA0012 are presented in Tab. 1.

Table 1. Freestream conditions and working gas of the micro-airfoil NACA0012cases

\begin{tabular}{|c|c|c|c|c|c|c|c|c|}
\hline Case & $p_{\infty}(\mathrm{Pa})$ & $M_{\infty}$ & $\mathbf{u}_{\infty}(\mathrm{m} / \mathrm{s})$ & $T_{\infty}(\mathrm{K})$ & $T_{w}(\mathrm{~K})$ & Gas & AOA (deg.) & $K n$ \\
\hline \multirow{3}{*}{ Airfoil [18] } & 2.78443 & 2 & 509 & 161 & 290 & Air & 0 and 10 & 0.026 \\
\cline { 2 - 9 } & 0.72395 & 2 & 509 & 161 & 290 & Air & 0 and 10 & 0.1 \\
\cline { 2 - 9 } & 0.27844 & 2 & 509 & 161 & 290 & Air & 20 & 0.26 \\
\hline
\end{tabular}

The profile of the NACA 0012 airfoil is computed by the expression below [23]

$$
y=0.6\left(0.2969\left(\frac{x}{c}\right)^{0.5}-0.126\left(\frac{x}{c}\right)-0.3537\left(\frac{x}{c}\right)^{2}+0.2843\left(\frac{x}{c}\right)^{3}-0.1015\left(\frac{x}{c}\right)^{4}\right),
$$

where $c$ is the chord length, $c=4 \mathrm{~cm} ; x$ is the running distance along the chord $(0 \leq$ $x \leq c) ; y$ is the half thickness of the airfoil. The dimensions of geometries and numerical 


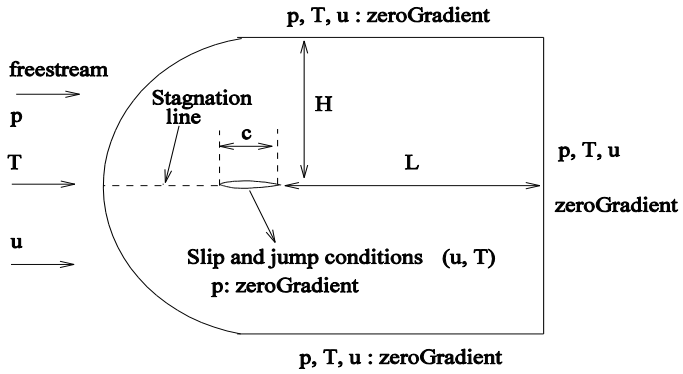

Fig. 2. Geometry, dimensions and numerical setups of the NACA 0012 airfoil case [18]

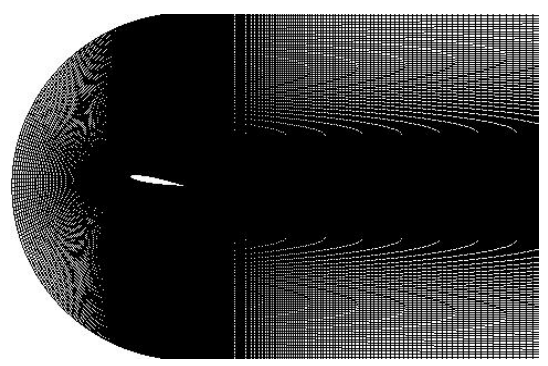

Fig. 3. Structured C-type mesh of the NACA 0012 airfoil case at $\mathrm{AOA}=10-\mathrm{deg}$. [18]

setup of computational domains of the airfoil case are presented in Fig. 2. The structured C-type mesh is used, as seen in Fig. 3, and the final mesh is adapted from previous work in $[17,18]$ with 600 cells on the airfoil surface, and the values $L=6 c, H=3 c$.

\section{SIMULATION RESULTS AND DISCUSSIONS}

Our CFD simulation results of the micro-airfoil cases would be compared with the DSMC and experimental data in [16-18]. DSMC is the best accurate method for simulating rarefied gas flows so far but its computational effort is quite expensive. We focus on the simulation results of the surface gas temperatures to evaluate our new temperature jump condition. Nonequilibrium boundary conditions are applied to the surface with $\sigma_{T}=\sigma_{u}=1.0$ for the CFD simulations. The accommodation coefficients of unity were also used for DSMC data.

\subsection{Cases $\mathrm{AOA}=\mathbf{0}-\mathrm{deg}, \mathrm{Kn}=\mathbf{0 . 0 2 6}$ and 0.1}

Figs. 4 and 5 present the surface gas temperatures over the airfoil surface of the cases $\mathrm{AOA}=0$-deg with $\mathrm{Kn}=0.026$ and 0.1 , respectively.

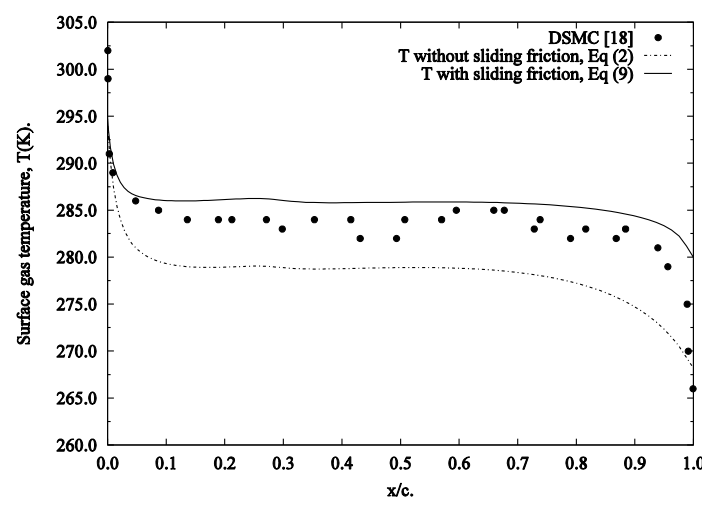

Fig. 4. Surface gas temperature distribution along the airfoil surface, $\mathrm{AOA}=0$-deg., $\mathrm{K} n=0.026$

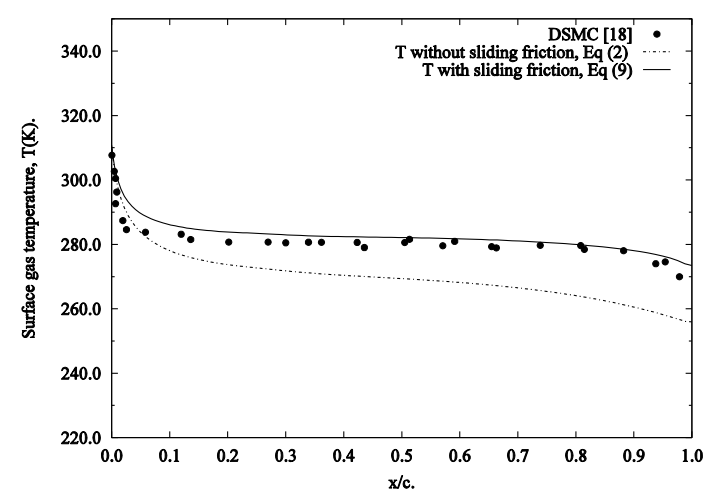

Fig. 5. Surface gas temperature distribution along the airfoil surface, $\mathrm{AOA}=0$-deg.,

$$
\mathrm{K} n=0.1
$$

Both of cases the temperatures reach the peak values at the airfoil tip, and thereafter gradually decrease along the surface. The surface gas temperatures with the sliding 
friction of two cases give good agreements with the DSMC data [18] while those without the sliding friction do not.

\subsection{Case $\mathrm{AOA}=10-\mathrm{deg}, \mathrm{Kn}=\mathbf{0 . 0 2 6}$}

The surface gas temperatures over the lower surface are presented in Fig. 6. At the airfoil tip, the simulations obtain the peak values of $294 \mathrm{~K}, 303 \mathrm{~K}$ and $292.5 \mathrm{~K}$ for temperatures with and without the sliding friction, and DSMC data $[17,18]$, respectively. The DSMC data and the temperature with the sliding friction slightly decrease to the location $x / c=0.05$. Past this location, the latter one is nearly a finite constant value to $x / c=0.9$, while the DSMC data oscillate in the range $0.05 \leq x / c \leq 0.9$. Both of them decrease in $0.9 \leq x / c \leq 1$. There is good agreement between the DSMC data and the temperature with the sliding friction. Past the airfoil tip the temperature without the sliding friction gradually decreases along the surface and underpredicts the temperature in the range $0.05 \leq x / c \leq 1$.

Fig. 7 shows the surface gas temperatures along the upper surface. They reach the peak values of 1) $292 \mathrm{~K}$ for the temperature with the sliding friction, 2) $301 \mathrm{~K}$ for that without the sliding friction, and 3) $290 \mathrm{~K}$ for DSMC data $[17,18]$. Past these peak values, the temperature with the sliding friction rapidly decreases until the location $x / c=0.05$, and then slightly increases along the upper surface. The DSMC data also reduce until the location $x / c=0.05$, and oscillate along the surface until the location $x / c=0.95$ before decreasing at the airfoil tail. The temperature without the sliding friction gradually decreases along the lower surface, and obtains the lowest values. The temperature with the sliding friction is close to DSMC data.

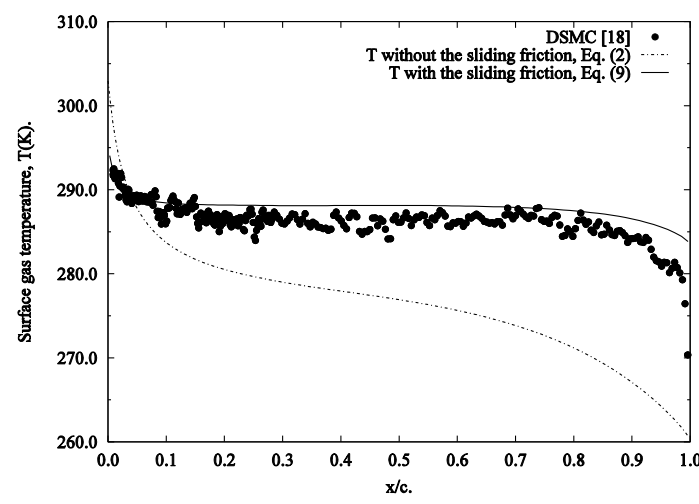

Fig. 6. Surface gas temperature distribution along the lower surface, $\mathrm{AOA}=10$-deg.,

$$
K n=0.026
$$

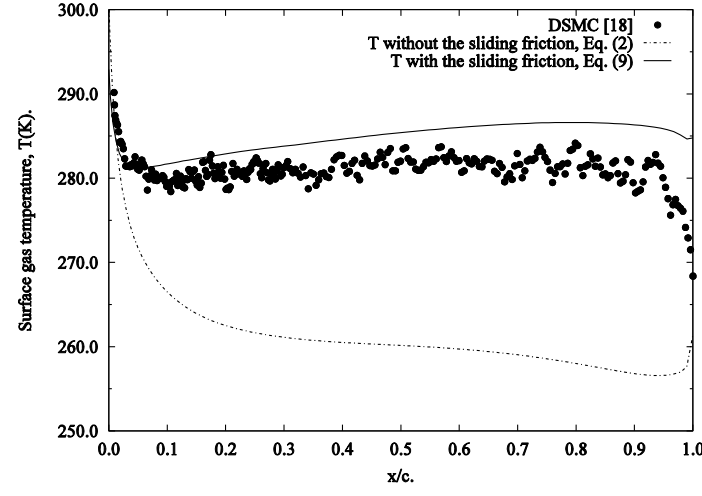

Fig. 7. Surface gas temperature distribution along the upper surface, $\mathrm{AOA}=10$-deg.,

$$
K n=0.026
$$

The normalized density contour $\left(\rho / \rho_{\infty}\right)$ of the CFD simulation with the new temperature jump condition is compared with experimental data [16], seen in Fig. 8. There is a relative agreement between the CFD results and experimental data for the slight rarefied gas at $K n=0.026$. 


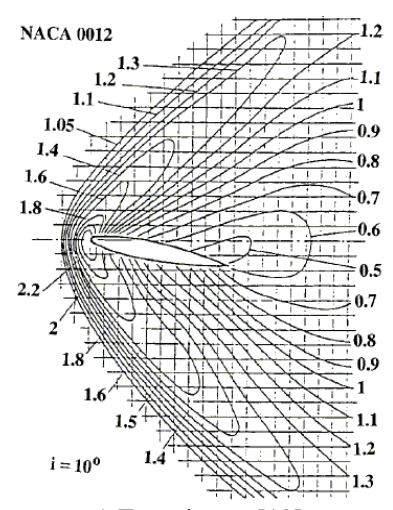

(a) Experiment [19]

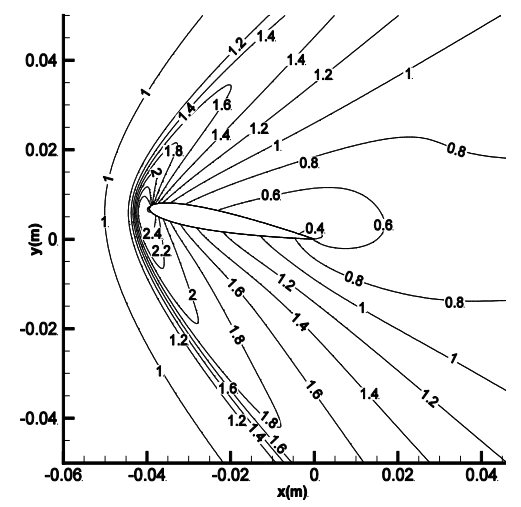

(b) Simulation with new temperature jump condition

Fig. 8. Normalized density contours $\left(\rho / \rho_{\infty}\right)$ of the case $M=2, \mathrm{AOA}=10-\mathrm{deg}, K n=0.026$

\subsection{Case $\mathrm{AOA}=10-\mathrm{deg}, \mathrm{Kn}=0.1$}

For the case $K n=0.1$, Fig. 9 presents the surface gas temperatures over the lower surface. The temperatures with and without the sliding friction reach the peak values of $301.5 \mathrm{~K}$ and $304 \mathrm{~K}$, respectively, while that of DSMC data is $295 \mathrm{~K}$. Past these peak values, the temperature with the sliding friction and DSMC data [17] gradually decrease along the lower surface. The temperature without the sliding friction underpredicts the surface temperature along the lower surface. There is a good agreement between the DSMC data and the temperature with the sliding friction.

The surface gas temperatures over the upper surface are shown in Fig. 10. All simulations obtain the peak values at the airfoil tip. All of them rapidly decrease to the location $x / c=0.08$. The DSMC data [17] oscillate in the range $0.8 \leq x / c \leq 1$, while the temperature with the sliding friction slightly decreases in the location $0.08 \leq x / c \leq 1$.

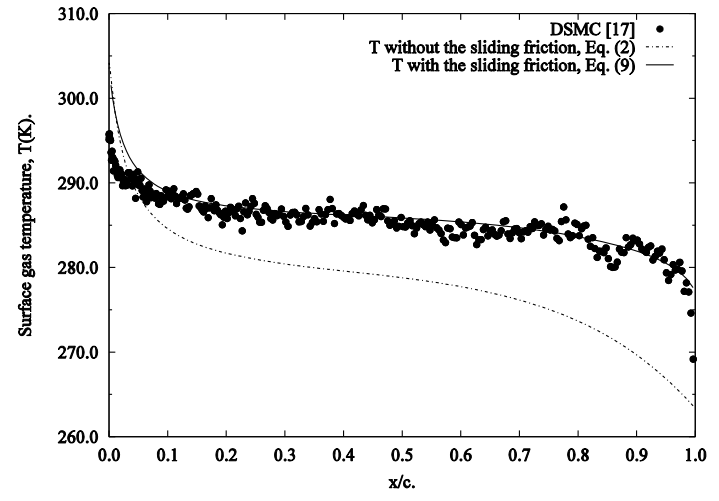

Fig. 9. Surface gas temperature distribution along the lower surface, $\mathrm{AOA}=10$-deg.,

$$
K n=0.1
$$

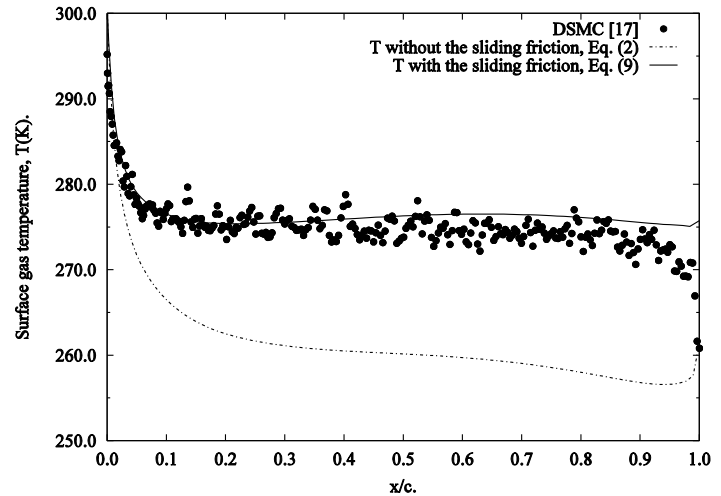

Fig. 10. Surface gas temperature distribution along the upper surface, $\mathrm{AOA}=10-\mathrm{deg}$,

$$
K n=0.1
$$


The temperature without the sliding friction is the lowest values along the upper surface. The DSMC data and the temperature with the sliding friction are close together.

\subsection{Case $\mathrm{AOA}=\mathbf{2 0}-\mathrm{deg}, \mathrm{Kn}=\mathbf{0 . 2 6}$}

Surface gas temperatures over the lower surface of the case $K n=0.26$, are presented in Fig. 11. All CFD and DSMC simulations obtain the peak values of 1) $301.2 \mathrm{~K}$ for the temperature with the sliding friction, 2) $301.4 \mathrm{~K}$ for that without the sliding friction, and 3) $297.5 \mathrm{~K}$ for the DSMC data [18], respectively at the airfoil tip. Past these peak values, the temperature with sliding friction and DSMC data gradually decrease along the lower surface while the temperature without the sliding friction very quickly reduces along the lower surface. The temperature with the sliding friction and DSMC data are close together along the surface. There is a large difference between the DSMC data and the temperature without the sliding friction.

Fig. 12 presents the surface gas temperatures over the upper surface. All simulation results reach the peak values of 1) $298.2 \mathrm{~K}$ for the temperature with the sliding friction, 2) 297.9K for that without the sliding friction, and 3) 294K for the DSMC data [17], respectively at the airfoil tip. Past these peak values both of the CFD results rapidly decrease until the location $x / c=0.274$. Thereafter they increase along the upper surface. The DSMC data gradually decrease until the location $x / c=0.25$, and then oscillate along the upper surface. The temperature with the sliding friction is better than that without the sliding friction in comparing with the DSMC data. Their average errors in comparing with the DSMC data are $2.31 \%$ and $2.73 \%$ for the temperatures with and without the sliding friction, respectively.

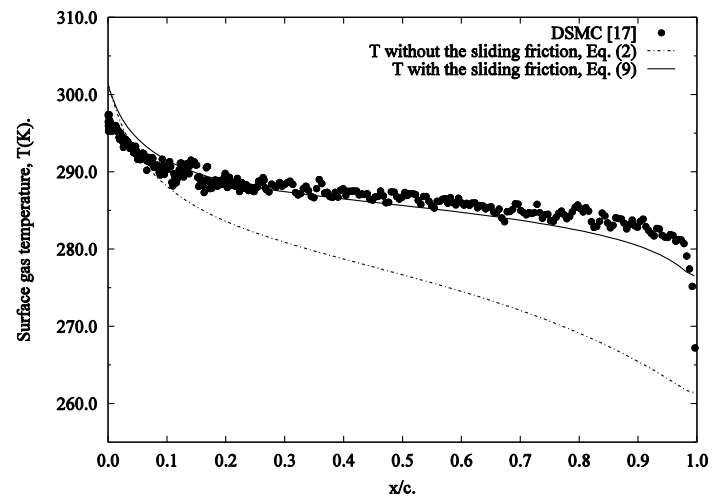

Fig. 11. Surface gas temperature distribution along the lower surface, $\mathrm{AOA}=20$-deg.,

$$
K n=0.26
$$

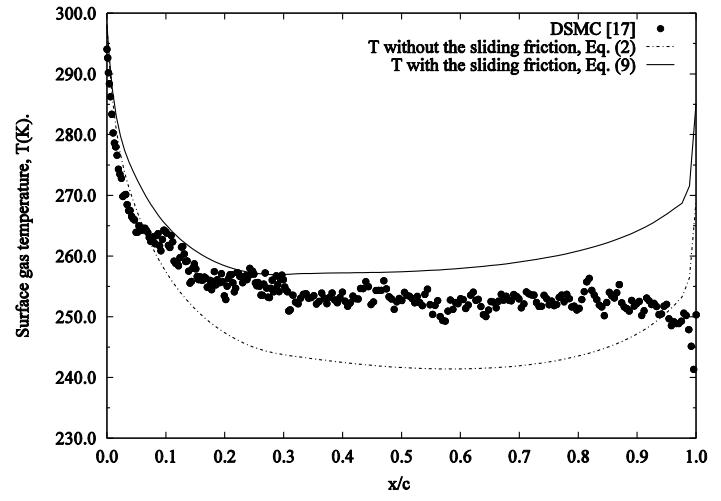

Fig. 12. Surface gas temperature distribution along the upper surface, $\mathrm{AOA}=20$-deg.,

$$
K n=0.26
$$

\subsection{Discussions}

It is seen that all surface gas temperature results with the new temperature jump can capture the DSMC data for all considered cases. This can be explained by the appearance of the slip velocity and the shear stresses in the new jump condition. These terms 
reduce the difference between the CFD and DSMC approaches in calculating the surface gas temperatures for the high Knudsen airfoil cases $(K n=0.1$ and 0.26$)$. In our previous CFD simulations of the NACA0012 micro-airfoil cases in [18], the shear stresses reached the peak values at the tip of the airfoil. Past these values, they gradually decrease along the airfoil surface. Corresponding to the shear stress distributions the slip velocities were slow due to viscous stress at the tip airfoil, and gradually increase to trailing edge of the airfoil. This results in the surface gas temperatures with the sliding friction are greater than those without the sliding friction. The simulation results in the present work figure out that the sliding friction of gas molecules over the surface is important in calculation of the surface gas temperature in the CFD method, and can reduce the gap of the simulation results of those between the CFD and DSMC method.

\section{CONCLUSIONS}

Our new temperature jump condition has been proposed by considering the sliding friction. The surface gas temperatures predicting by our new temperature jump condition give good agreements with the DSMC data while those predicting by the jump condition without the sliding friction do not, especially the NACA0012 micro-airfoil cases with the high Knudsen numbers, $K n=0.1$ and $K n=0.26$ with AOA $=20$-deg. It also figured out that since the slip velocity is applied to the surfaces in the CFD method then the sliding friction must be considered in the derivation of the temperature jump condition. From simulation results obtained in the present work, our new jump condition should be an alternative temperature jump condition for prediction of the surface gas temperature in CFD.

\section{ACKNOWLEDGEMENTS}

This research is funded by Vietnam National Foundation for Science and Technology Development (NAFOSTED), under grant number 107.03-2015.16.

\section{REFERENCES}

[1] M. von Smoluchowski. Über wärmeleitung in verdünnten gasen. Annalender Physik und Chemie, 64, (1898), pp. 101-130.

[2] A. Q. Zade, M. Renksizbulut, and J. Friedman. Boundary conditions for multi-component slip-flows based on the kinetic theory of gases. In Proceeding of ASME 2008 6th International Conference on Nanochannels, Microchannels, and Minichannels. American Society of Mechanical Engineers, (2008), pp. 75-83. doi:10.1115/icnmm2008-62178.

[3] T. Gökçen and R. W. MacCormack. Nonequilibrium effects for hypersonic transitional flows using continuum approach. Technical Report 461, AIAA paper, (1989). doi:10.2514/6.1989461.

[4] R. S. Myong. Gaseous slip models based on the Langmuir adsorption isotherm. Physics of Fluids, 16, (1), (2004), pp. 104-117. doi:10.1063/1.1630799.

[5] N. T. P. Le, C. White, J. M. Reese, and R. S. Myong. Langmuir-Maxwell and LangmuirSmoluchowski boundary conditions for thermal gas flow simulations in hypersonic aerodynamics. International Journal of Heat and Mass Transfer, 55, (19), (2012), pp. 5032-5043. doi:10.1016/j.ijheatmasstransfer.2012.04.050. 
[6] R. G. Deissler. An analysis of second-order slip flow and temperature-jump boundary conditions for rarefied gases. International Journal of Heat and Mass Transfer, 7, (6), (1964), pp. 681694. doi:10.1016/0017-9310(64)90161-9.

[7] N. T. P. Le and E. Roohi. A new form of the second-order temperature jump boundary condition for the low-speed nanoscale and hypersonic rarefied gas flow simulations. International Journal of Thermal Sciences, 98, (2015), pp. 51-59. doi:10.1016/j.ijthermalsci.2015.06.017.

[8] J. Van Rij, T. Ameel, and T. Harman. The effect of viscous dissipation and rarefaction on rectangular microchannel convective heat transfer. International Journal of Thermal Sciences, 48, (2), (2009), pp. 271-281. doi:10.1016/j.ijthermalsci.2008.07.010.

[9] D. A. Lockerby, J. M. Reese, D. R. Emerson, and R. W. Barber. Velocity boundary condition at solid walls in rarefied gas calculations. Physical Review E, 70, (1), (2004), p. 017303. doi:10.1103/physreve.70.017303.

[10] S. H. Maslen. On heat transfer in slip flow. Journal of the Aerospace Sciences, (2012). doi:10.2514/8.7698.

[11] J. A. Bartz and R. J. Vidal. Surface measurements on sharp flat plates and wedges in lowdensity hypersonic flow. AIAA Journal, 7, (6), (1969), pp. 1099-1109. doi:10.2514/3.5281.

[12] N. T. P. Le, N. A. Vu, and L. T. Loc. Effect of the sliding friction on heat transfer in highspeed rarefied gas flow simulations in cfd. International Journal of Thermal Sciences, 109, (2016), pp. 334-341. doi:10.1016/j.ijthermalsci.2016.06.020.

[13] A. J. Lofthouse, L. C. Scalabrin, and I. D. Boyd. Velocity slip and temperature jump in hypersonic aerothermodynamics. Journal of Thermophysics and Heat Transfer, 22, (1), (2008), pp. 3849. doi:10.2514/1.31280.

[14] G. A. Bird. The DSMC Method. Clarendon, Oxford, (2013).

[15] OpenFOAM. http://www.openfoam.org, (August, 2016).

[16] J. Fan, I. D. Boyd, C.-P. Cai, K. Hennighausen, and G. V. Candler. Computation of rarefied gas flows around a NACA 0012 airfoil. AIAA Journal, 39, (4), (2001), pp. 618-625. doi:10.2514/2.1384.

[17] A. Shoja-Sani, E. Roohi, M. Kahrom, and S. Stefanov. Investigation of aerodynamic characteristics of rarefied flow around NACA 0012 airfoil using DSMC and NS solvers. European Journal of Mechanics-B/Fluids, 48, (2014), pp. 59-74. doi:10.1016/j.euromechflu.2014.04.008.

[18] N. T. P. Le, A. Shoja-Sani, and E. Roohi. Rarefied gas flow simulations of NACA 0012 airfoil and sharp 25-55-deg biconic subject to high order nonequilibrium boundary conditions in CFD. Aerospace Science and Technology, 41, (2015), pp. 274-288. doi:10.1016/j.ast.2014.12.019.

[19] J. C. Maxwell. On stresses in rarified gases arising from inequalities of temperature. Philosophical Transactions of the Royal Society of London, 170, (1879), pp. 231-256. doi:10.1017/cbo9780511710377.068.

[20] C. Hong and Y. Asako. Some considerations on thermal boundary condition of slip flow. International Journal of Heat and Mass Transfer, 53, (15), (2010), pp. 3075-3079. doi:10.1016/j.ijheatmasstransfer.2010.03.020.

[21] E. H. Kennard. Kinetic theory of gases. McGraw-Hill Book Company Inc, (1938). doi:10.1038/142494a0.

[22] C. J. Greenshields, H. G. Weller, L. Gasparini, and J. M. Reese. Non-oscillatory central schemes for high speed viscous flows. International Journal for Numerical Methods in Fluids, 23, (2010), pp. 1-21.

[23] I. H. Abbott and A. E. Von Doenhoff. Theory of wing sections, including a summary of airfoil data. McGraw Hill, (1949). 